\title{
A Computing System to Assist Business Leaders in Making Ethical Decisions
}

\author{
Reggie Davidrajuh \\ University of Stavanger Department of \\ Electrical Engineering and Computer Science \\ PO Box 8002, Norway \\ Email: reggie.davidrajuh@uis.no
}

\begin{abstract}
This paper explores whether it is possible to build a computing system that can make ethical decisions autonomously, and if it is possible, then what it takes to build such a system. Firstly, this paper introduces ethical business decision-making, and also explains the reason for building an autonomous computing system that can assist business leaders. Secondly, a literature study is presented on the existing models for ethical decision-making; from the literature study, and with the help the stakeholder analysis (ethical theories that are relevant to the business environment), a new model is proposed. Thirdly, based on the new model, this paper proposes building an autonomous computing system; the proposed system has a layered architecture. This paper concludes that if such a system is built then inherently it has to be an adaptive system in order to cope with ever changing environment.
\end{abstract}

\section{Introduction}

The collapses of Enron, WorldCom, Arthur Andersen, Martha Stewart's stock sales, etc. have made us aware of the seriousness of ethical implications of business decisions. These days, business decision makers must incorporate ethics in their business decisions. However, confronting ethical dilemmas and making ethical decisions are not easy as:

- There are no magic formulas available to help the decision makers solving ethical dilemmas they confront

Please use the following format when citing this chapter:

Davidrajuh, R., 2008, in IFIP International Federation for Information Processing, Volume 286; Towards Sustainable Society on Ubiquitous Networks, eds. Oya, M., Uda, R., Yasunobu, C., (Boston: Springer), pp. 303-314. 
- When confronting ethical issues, huge number of variables (from sociology, psychology, economics, business, laws \& regulations, etc.) that have to be considered. Hence, without any computing aid, it is not easy for decision makers to make an 'optimal' solution

Thus, this paper proposes an autonomous system to help decision makers incorporate ethics in their business decisions. In order to develop such a system:

Firstly, it is necessary to get a systems perspective of ethical business decisionmaking in the networked economy: what are the elements and environments involved in the decision-making process, how the elements are connected or related to each other, how the elements, environments, and the interconnections can influence each other, etc.

Secondly, it is necessary to devise a model for the autonomous computing system for decision-making.

Thirdly, a validation of the model has to be done; whether suitable enabling technology is available to realize such a system? Will it be possible to program the system? Etc.

\section{Why a Computing System?}

Despite the growing interest in ethical decision-making, there is considerable disagreement about the appropriate way to define business ethics, and business ethical leadership, and the ways to asses the ethical decisions (Yukl, 2006; Heifetz, 1994).

Generally, ethical business decision making is such a difficult process, so much that business leaders use their moral standards to evaluate their ethical decisions as good or bad depending on what extend to which the outcomes of their decisions violate basic laws of society, denies others their rights, endangers the health and lives of other people, or involves attempts to deceive and exploit others for personal benefits. This paper proposes implementing business ethical decision-making processes as computer software so that it can help solving the following problems associated with ethical decision-making:

Ambiguous process: Ethical decision-making is an ambiguous process that appears to include a huge number of highly interconnected Webs of sub-processes. This is due to the existence of several criteria that are relevant for judging ethical decisions, including the person's values, the person's stage of moral development, conscious intentions, freedom of choice, use of ethical and unethical behavior, and the probable outcomes of the ethical decisions (Yuk1, 2006).

Dependency on moral development: Kohlberg (1984) proposed a model to describe how people progress through six sequential stages of cognitive moral development as they grow from child to an adult. With each successive stage, the person develops a broader understanding of the principles of justice, social responsibility, and human rights. Unlike physical maturation, moral development is not inevitable, and some people become fixated at a particular stage. A leader who is a a higher level of development is usually regarded as more ethical than one at a lower level of development; the level of moral development of leaders has an impact on ethical decisionmaking in business organizations (Trevino, 1986; Trevino and Youngblood, 1990). 
Uncertainty in problem identification: An important leadership function is to help frame problems by clarifying key issues, encouraging dissenting views, distinguishing cases from symptoms, and identifying complex interdependencies (Yukl, 2006). In ethical decision-making, identifying and acknowledging key problems and issues is no easy task; a computer program may facilitate leaders systematically identify problems, acknowledge, delegate to followers, and solve problems.

Environmental influences: Ethical behavior occurs in a social context and it can be strongly influenced by aspects of the situation (Trevino, 1986; Trevino et al, 1998). Business leaders' personality and cognitive moral development interact with aspects of the situation in the ethical decision-making. That is, ethical decisions can be explained better by consideration of both the individual and the situation than either variable alone (Yukl, 2006).

Formal Assurance: Burns (1978) and Heifetz (1994) describe leadership as both a dyadic and collective process. Leaders influence individuals, and they also mobilize collective efforts to accomplish adaptive work. The type of influence used by leaders includes not only use of rationality and appeal to values, but also formal authority. Leaders can use their authority to direct attentions to problems, frame issues, structure decision processes, mediate conflicts, allocate resources to support problem solving, and delegate specific responsibilities to individuals or groups. Though formal authority is not necessary as emergent leaders acquire informal authority by taking responsibility for exercising leadership institutions where it is needed, Heifetz (1994) emphasizes that meaningful change requires shared leadership, and it can not be accomplished by a single, heroic individual. A significant and formal assurance from a computer program could function as a solid backing to leaders to put into practice their decisions.

Empirical research on ethical issue in leadership is relatively new topic, and much still needs to be learned about it (Yukl, 2006). Kahn (1990) proposed an agenda of research questions that would help to bridge the apparent gap between normative concepts (defining ethical behavior) and contextual concepts (the conditions influencing ethical behavior). The objective is to produce knowledge that strengthens both the theory and practice of ethical conduct in Organizations. Examples of relevant research questions include language used to frame and communicate ethical issues, the conditions under which conversations about ethics are likely to occur, the process by which ethical dilemmas and disagreements are resolved, the process by which ethical principles are adapted to changing conditions, and the ways that leaders influence ethical awareness, dialogue, and consensus.

This paper proposes a natural extension to the line of thought of Kahn (1990); the proposal is to implement the process of ethical business decision-making as a computing system, so that a systematic analysis of the process can be done.

\section{Modeling Ethical Decision-Making}

First, a literature study is given on the existing models for ethical decision-making in the networked economy. From the literature study, a new model for ethical decision-making is developed. 


\subsection{Existing Models for Ethical Decision-Making}

\subsubsection{A Model Based on Four Constraints}

In business environments, there are many constraints that can guide and shape business transactions. Lessig (1999) presents a model describing four constraints that regulate the ethical behavior of cyberspace activities.

The first constraint is the law. Laws are rules or commands imposed by the government that are enforced through ex post sanctions; ex post sanction means that law retroactively makes criminal conduct not criminal when performed, but increases the punishment for crimes already committed. The second constraint is the market. The market regulates through the price it sets for goods and services.

The third constraint is the code ( $a k a$ architectural constraint). The architectural constraints are physical constraints, natural or man-made, restricts the freedom of business transactions. The fourth constraint is the social norms. Social norms are informal expressions of a community that defines a well-defined sense of normalcy and expects the members of the community to follow. An example for social norm under business context is the dress code.

\subsubsection{Modified Model by Spinello}

The model by Lessig (1999) incorporated ethics under the broad category of "social norms"; social norms have only cultural or community value. Spinello (2003) argues that the fundamental principles of ethics are metanorms and they have universal validity, and hence should not be classified as social norms. In the modified model by Spinello, ethics is given a directive role, that is, ethics should guide and direct the ways in which the constraints such as laws, the market, code, and social norms, exercise their regulatory power.

\subsubsection{A Model Based on Six Environments}

Walstrom (2006) conducted an empirical study to investigate factors that impact on ethical decision-making processes regarding information ethics. Walstrom (2006) found that the two factors that had predominant impact were:

- The social environment: religious values, cultural values, and social values

- The government/legal environment: legislation, administrative agencies, judicial systems, etc.

However, there exist four other factors too that exercise influence on ethical decision-making (Boomer et al, 1987):

- Personal environment: individual attributes including personal goals, motivation, position, demography, 
- Private environment: peer group, family, and their influences,

- Professional environment: code of conduct, professional meetings, licensing,

- Work environment: corporate goals, stated policy, corporate culture.

\subsubsection{A Model Emphasizing Personal Environment}

On contrary to the model by Walstrom (2006) that is based on six environments emphasizing social and legal environments, Haines and Leonard (2007) suggests that the impact of the personal and private environments have a greater influence in certain ethical problems.

\subsection{Theoretical Basis for Developing a New Model}

It is easy to see that the existing models presented in the previous subsection are only for qualitative reasoning; and that these models can not be used towards realization of computer systems that can make autonomous decisions, as the models do not facilitate inclusion of mathematical modules for computation. Thus, in this subsection, a new model for ethical decision-making is developed; the main reason for developing the new model is to build a computing system that can autonomously make ethical decisions.

Although there are no magic formulas to start with, it is helpful to have a framework with which the ethical decision-making process can be organized (Silbiger, 2007); stakeholder analysis is a framework that helps us identify various elements involved in the decisions.

\subsubsection{Stakeholder Analysis}

Under stakeholder analysis, three theories of ethics are applied in business environments. These are stockholder theory, stakeholder theory, and social contract theory. These theories and their interpretations and implications are given below:

Stockholder Theory: According to the stockholder theory, the stockholders contribute capital to the businesses; corporate leaders act as agents in advancing the stockholders interests (Pearlson and Saunders, 2006). According to the originator of this theory, the only social responsibility of business and hence the agents, is to use the resources to engage in business activities designed to increase profits for the stockholders; profit making must be done by open and free competition, without deception or fraud (Friedman, 1962; Pearlson and Saunders, 2006).

Stakeholder Theory: According to the Stakeholder theory, in addition to the obligation to the stockholder, agents are also responsible for taking care of the interests of all the stakeholders of the business; the term stakeholder refers to any group that vitally affects the survival and success of the corporation (e.g. employees, suppliers, distributors, customers) or whose interest the corporation vitally affects (e.g. the local community, customers) (Smith and Hasnas, 1999). This means, unlike stockholder 
theory that primarily look into the interests of stockholders, stakeholder theory balances the rights of all stakeholders (Pearlson and Saunders, 2006).

Social Contract Theory: Both stockholder theory and stakeholder theory do not talk about the society; according to the social contract theory, agents are responsible for taking care of the needs of a society without thinking about corporate or other complex business arrangements. Social contract theory forces the agents to interact in a way that business transactions bring benefits to the members of a society. Hence, society can grant legal recognition ('social contract') to a corporation to allow it to employ social resources toward given ends (Smith and Hasnas, 1999). The social contract allows a corporation to exist and demands that agents create more value to the society than they consume for the business transactions.

Summary of Stakeholder Analysis: By skimming through the three theories of business ethics, one can see that these three theories related. The social contract theory is the most restrictive one, demanding that the whole society should be taken care of by the agents when they conduct business exchanges. The stakeholder theory is lesser restrictive than the social contract theory, as instead it demands that all the stakeholders of the business (not the whole society) should be taken care of. Finally, the stockholder theory is the least restrictive one, as it demands that only the stockholders are to be taken care of by the agents. In summary, stakeholder analysis presented above suggests that first we draw a list of all the elements (stockholders, customers, etc.) potentially effected by an ethical decision; then, we evaluate net economic benefits that the ethical decision will cause on each elements on the list.

\subsection{The New Model}

Based on the stakeholder analysis presented in section 2 and on the literature study on the existing models for ethical decision-making, presented in section 3 , we formulate a new model consisting of the following processes:

Initialization: Identifying the main elements (stakeholders) and the environments involved in the ethical issue.

Establishing the connected system: Determining the rights and responsibilities of each element and the relative weights of each element, thus establishing the connection between the elements.

The process of measurement: Setting up the governing equations that that combines the elements and the environments, and measuring the harms and benefits to each element, and finally, making decisions based on the net harms and benefits to the elements involved in the issue.

\subsubsection{Identifying the Primitive Elements of the Model}

There are a number of elements already identified in the literature: Lessig (1999) identifies four elements such as laws, the market, code, and social norms, as the primitive elements of a system for ethical business decision-making. Walstrom (2006) identifies six elements such as social environment, legal (or government) environ- 


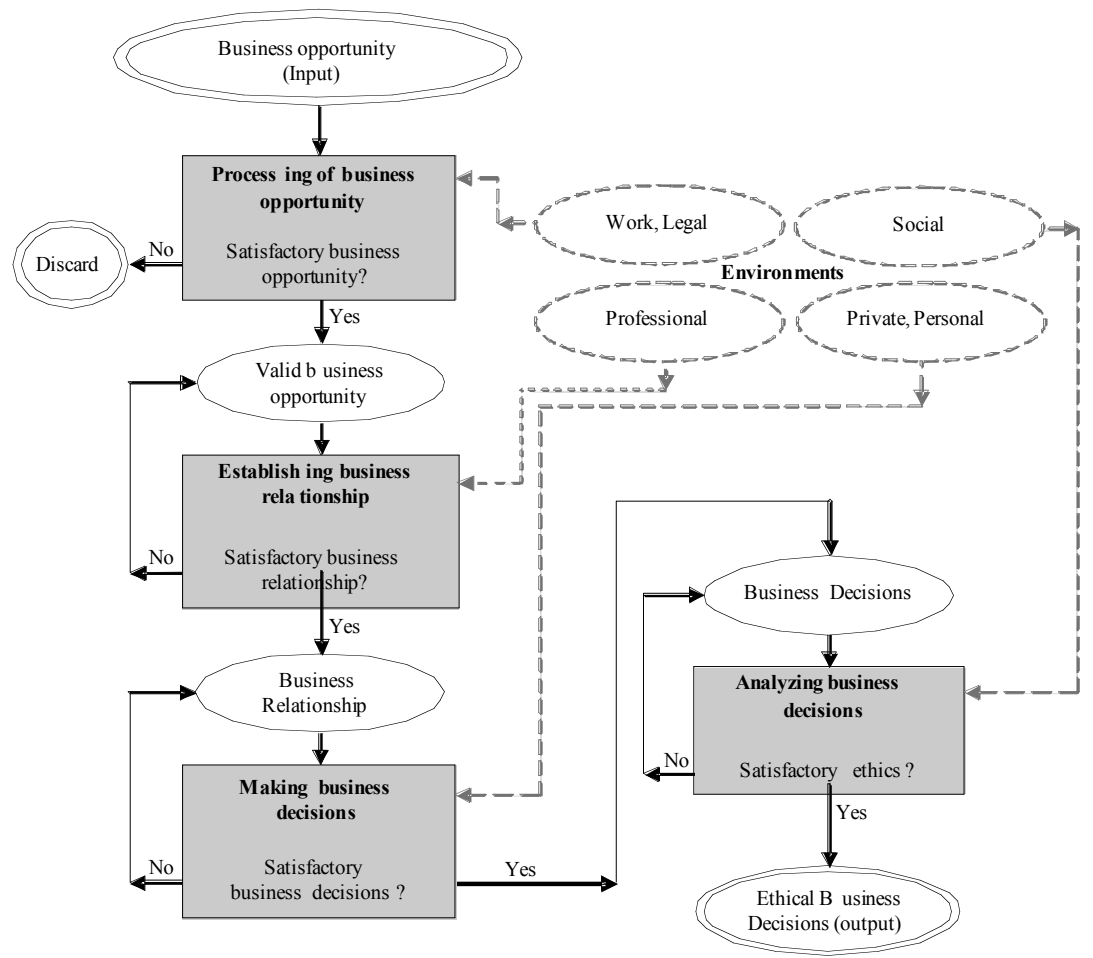

Figure 1: State flow diagram for ethical business decisi on-making

ment, personal environment, private environment, professional environment, and work environment, as the primitive elements. In addition to all these elements, the literature also sites the following primitive elements: interacting agents, leaders, shareholders, etc.

\subsubsection{Establishing the Connections in the Model}

Before we start thinking about the internal connections of the system, let us identify the sources (or the external disturbances that agitate the system to produce an output) and the output of the system. Business opportunities are the sources of the system. Obviously, without business opportunities there won't be any ethical business decisions; ethical business decisions are the output of the system.

Given below is a step-by-step formulation of the connections between the primitive elements of the system:

When the input (a business opportunity) is fed into the systems, the legal environment and the work environment (business goals and objectives, etc.) must recognize the business opportunity as a valid one. For example, when a company in US receives a business opportunity from a company in Cuba, the legal environment will reject the opportunity. In some other cases, an opportunity may be rejected because the oppor- 
tunity does not satisfy business goals and objectives (work environment) of a company.

Business relationships evolve from valid business opportunities, to realize business exchanges. The business relationships are formulated by the professional environment (code of conduct, professional meetings, etc.) of the respective companies involved.

Business decisions are made to strengthen profits from the business relationships. A major player that influence formulation of business decisions for business relations is the personal environment (individual attributes including personal goals, motivation, position, etc.) and the private environment (peer group inclusive colleagues and immediate leaders, family and their influences).

Finally, ethical business decisions evolve from business decisions. As Walstrom (2006) states, social environment (religious values, cultural values, and social values) plays the major role in shaping ethical business decisions. In addition, the agent's personal ethics (might also be called morality - the ability to recognize moral issues, make moral judgment, awareness about profit for "all the stakeholders", etc.) play en important role.

Figure 1 shows the state flow diagram for the ethical business decision-making processes. As figure 1 depicts, business goals and objectives are the driving force of business relationships, which is opened up by business opportunities. The six socio economic environments formulate the business decisions. And finally, it is the agent's moral judgment that shapes the business decisions; the agent's moral judgment depends on his or hers ability to recognize the moral issues, to establish moral intent, engagement in moral behavior, characteristics of the moral issue, and the individual's own characteristics or personality (Haines and Leonard, 2007).

\section{Developing the Proposed Computing System}

The architecture of the computing system is a hybrid architecture based on previous works on autonomous and adaptive business systems; se Muller et al (1995), Fasli (2007), and Woolridge (1999) for some of the architectures. The architecture consists of three distinguishable layers, such as Planning layer, Inference layer, and Data layer.

\subsection{Planning Layer}

The planning layer uses the models to predict potential outcome of a scenario. First it checks the overall validity of the business opportunity (see figure-1); it then generates goals which are associated with ethical business strategies. Subsequently, business goals are propagated down to the inference layer, which uses the data layer to make decisions. The inference layer hosts a number of inference engines. The data layer mainly consists of a knowledge base. 


\subsection{Inference Layer}

In figure 1, oval shaped components are passive components (such as input buffers for incoming business opportunities, intermediate buffers for storing intermediate decisions made, and output buffers for storing final decisions, etc). Rectangular components are active components, such as inference engines for decision-making. In figure-1, four inference engines are visible: 1) Processing of business opportunity, 2) Establishing business relationships, 3) Making business decisions, and 4) Analyzing business decisions. These inference engines are the main components of the inference layer.

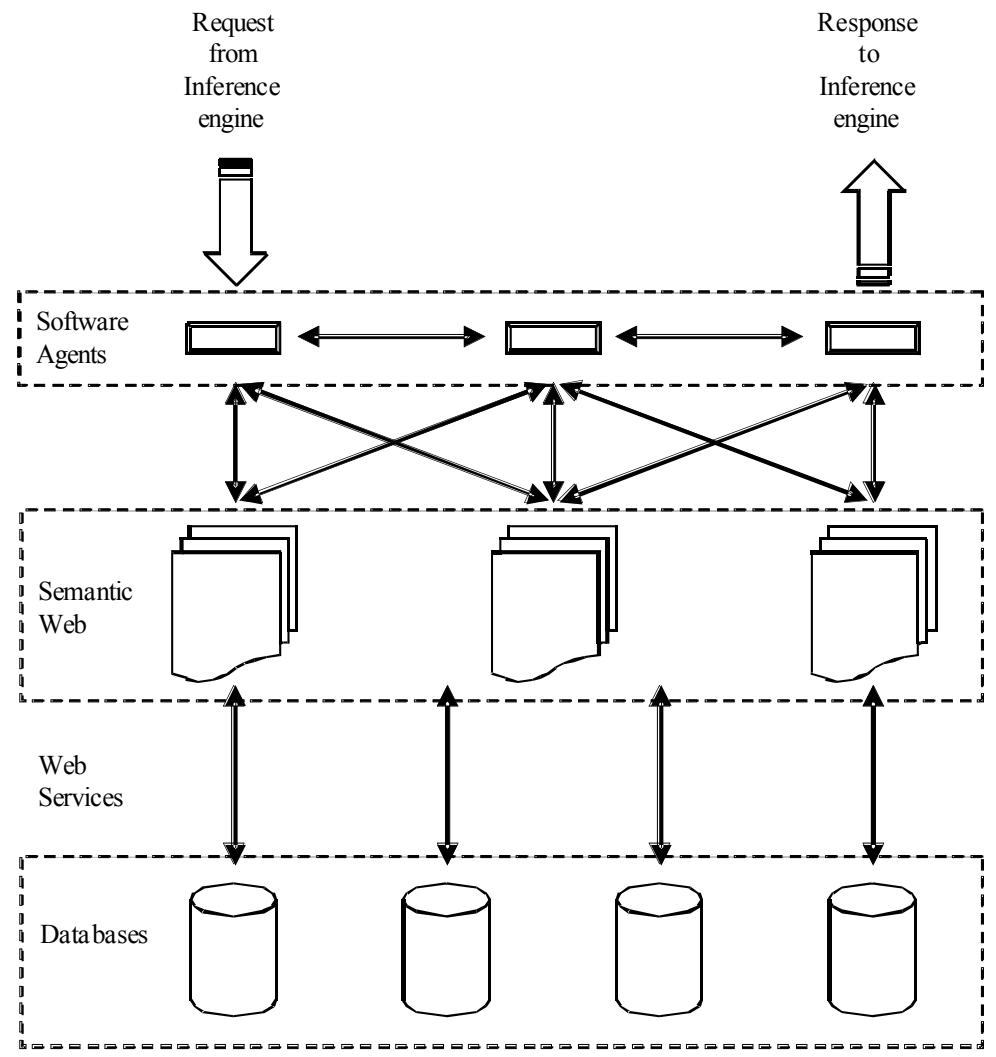

Figure-2: The data layer that consists of many brokers working together

The inference engines are equipped with mathematical models for decisionmaking. We believe that it is possible establish mathematical models to measure net economic benefits even for the complex problems like ethical issues, as the necessary enabling technologies are already available. We can utilize fuzzy logic (Ross, 2004; Tsoukalas and Uhrig, 1997) to code the mathematical models; the reason for propos- 
ing the use fuzzy logic is that fuzzy logic filters away inaccuracies in the input parameters; in addition, compared to pure mathematical approaches (such as mixed integer programming, linear programming, etc), with fuzzy logic it is easy to model a system.

\subsection{Data Layer}

Figure-2 shows details of the data layer, which consists of several brokers:

The software agents

Semantic Web

Web Services

Databases

At the bottom of the data layer rests data bases that are frequently updated to synchronize with the changes occurring in the external world. There can be many databases (e.g. as shown in figure-1, a database for storing data from each environment, legal database, work environment database, etc.). Since the databases are geographically distributed (it is less likely that any two databases are kept in the same location), Web services are used to delegate the data whenever needed.

Data from databases through Web services are pieces of data. We need ontologies to integrate the data together to form meaningful information. Finally, when a request comes from inference engine, it is the software agents that identify the needs, locate the Web services and delegate the response back to the inference engine.

\section{Concluding Remarks}

Leaders can do many things to promote ethical practices in organizations. The leader's own actions provide an example of ethical behavior to be imitated by people who admire and identify with the leader. Leaders can also set clear standard and guidelines for dealing with ethical issues, provide opportunities for people to get advice about dealing with ethical issues, and initiate discussions about ethical issues to make them more salient. However, as the section 2 explained, how can a leader be sure about whether the decisions he or she proposes are ethical or not? There are too many parameters involved, and one man ethically valid decisions is other man's unethical decision. This paper proposes an autonomous computing system to assist leaders making ethical decision.

The system proposed in figure- 2 should not be assumed as a static system as it may looks like. The databases shown in the figure are static databases, but frequently updated by a set of software agents that can learn about the environment, and the changes in the environment. Use of software agents gives the autonomous property to the proposed computing system. 


\section{References}

Boomer, M., Clarence, G., and Tuttle, M. (1987) A behavioral Model of Ethical and Unethical decision Making. Journal of Business Ethics, 6(4), (May 1987), pp. 265-280

Burns, J. (1978) Leadership. New York: Harper \& Row

Cassandras, G. and LaFortune, S. (1999). Introduction to Discrete Event Systems. Hague, Kluwer Academic Publications

Dickson, M., Smith, D. Grojean, M., and Ehrhart, M. (2001) An organizational climate regarding ethics: The outcome of leader values and the practices that reflect them. Leadership Quarterly, 12, 197-217

Fasli, M. (2007) Agent Technology for e-commerce. Wiley

Friedman, M. (1962) Capitalism and Freedom. Chicago: University of Chicago Press

Haines, R. and Leonard, L. (2007). Individual characteristics and ethical decision-making in an IT context. Industrial Management \& Data Systems, 107 (1), pp. 5-21

Heifetz, R. (1994) Leadership without easy answers. Cambridge, MA: Belnap Press of Harvard University Press

Kahn, W (1990) Toward an agenda for business ethics research. Academy of Management Review, $15,311-328$

Kohlberg, L. (1984) The psychology of moral development. New York: Harper \& Row

Lessig, L. (1999) Code and Other Laws of Cyberspace. New York: Basic Books

Muller, J., Pischel, M., and Thiel, M. (1995) Modelling reactive behaviour in vertically layered agent architecture. In Wooldridge, M. and Jennings, N., editors, Intelligent Agents: Agent Theories, Architectures, and Languages (ATAL), LNAI Volume 890, pages 261-276, Springer, Berlin

Pearlson, K. and Saunders, C. (2006) Managing \& Using Information Systems: A strategic Approach. 3ed., Wiley

Peterson, J. (1981) Petri Net Theory and the Modeling of Systems. Prentice-Hall, N.J.

Ross, T. (2004). Fuzzy logic with Engineering Applications. 2. ed. John Wiley \& Sons

Silbiger, S. (2007). The 10-Day MBA. Piatkus, London.

Smith, H. And Hasnas, J. (1999) Ethics and Information Systems: The Corporate Domain. MIS Quarterly, 23 (1), (Mar. 1999), pp. 109-127

Spinello, R. (2003) CyberEthics: Morality and Law in Cyberspace. 2nd ed. Jones and Bartlett Publishers

Trevino, L. (1986) Ethical decision making in organizations: A person-situation interactionist model. Academy of Management Review, 11, 601-617

Trevino, L. Butterfield, K. and McCabe, D. (1998) The ethical context in organizations: Influences on employee attitudes and behaviors. Business Ethics Quarterly, 8(3), 447-476 
314 Reggie Davidrajuh

Trevino, L. and Youngblood, S. (1990) Bad apples in bad barrels: a casual approach. Journal of Applied Psychology, 75, 378-385

Tsoukalas L. and Uhrig, R. (1997). Fuzzy and Neural Approaches in Engineering. John Wiley and Sons

Walstrom, K (2006) Social and legal impacts on information ethics decision making. Journal of Computer Information Systems, XLVII (2), (Winter 2006-2007), pp. 1-8

Wooldridge, M. (1999) Intelligent Agents. In Weiss, G., editor, Multiagent Systems: A modern approach to Distributed Artificial Intelligence, pages 27-77. The MIT Press, Cambridge, MA

Yukl, G. (2006) Leadership in organizations. 6th Ed. Pearson / Prentice-Hall. 